\title{
Enhancement of Coulomb interactions in semiconductor nanostructures by dielectric confinement
}

\author{
G. Goldoni $^{1,2}$, F. Rossi ${ }^{1,3}$, A. Orlandi ${ }^{1,2}$, M. Rontani ${ }^{1,2}$, F. Manghi ${ }^{1,2}$, E. Molinari ${ }^{1,2}$ \\ ${ }^{1}$ Istituto Nazionale per la Fisica della Materia (INFM) \\ ${ }^{2}$ Dipartimento di Fisica, Università di Modena, Via Campi 213/A, I-41100 Modena, Italy \\ 3 Dipartimento di Fisica, Politecnico di Torino, C.so Duca degli Abruzzi 24, I-10129 Torino, Italy
}

(November 7, 2017)

\begin{abstract}
We present a theoretical analysis of the effect of dielectric confinement on the Coulomb interaction in dielectrically modulated quantum structures. We discuss the implications of the strong enhancement of the electron-hole and electron-electron coupling for two specific examples: (i) GaAs-based quantum wires with remote oxide barriers, where combined quantum and dielectric confinements are predicted to lead to room temperature exciton binding, and (ii) semiconductor quantum dots in colloidal environments, where the manybody ground states and the addition spectra are predicted to be drastically altered by the dielectric environment.
\end{abstract}

When a semiconductor nanostructure is embedded in a medium with a smaller dielectric constant, the Coulomb interaction between quantum confined states may be enhanced by virtue of the polarization charges which form at the dielectrically mismatched interfaces ${ }^{0}$. While this effect is relatively small and usually neglected in conventional semiconductor heterostructures (e.g., GaAs/AlAs), we will show that, for hybrid semiconductor nanostructures surrounded by an organic or dielectric medium the enhancement can be large and must be taken into account for a realistic description of Coulomb correlated quantum states. Beside being quantitatively important for the interpretation of experimental spectra, these effects provide an additional degree of freedom for tailoring optical and transport properties of quantum structures.

In this paper we examine two prototype examples relevant to the physics of quantum wires (QWIs) and dots (QDs). We first consider properly designed hybrid semiconductor/insulator QWIs based on GaAs, and show that dielectric confinement (DC) may lead to excitonic states with a binding energy exceeding the room temperature thermal energy $k T_{\text {room }}$ (a prerequisite for exploiting excitonic states in electro-optical devices) without degrading the optical efficiency typical of conventional GaAs/AlAs nanostructures. Secondly, we show that the dielectric constant of the environment may strongly affect the addition spectra of QDs by modifying the electronic ground state with respect to the case of good dielectric matching.

Our theoretical schemel moves from the following basic considerations. When the dielectric constant $\epsilon(\mathbf{r})$ is spatially modulated, the Coulomb interaction between, say, two electrons sitting at positions $\mathbf{r}$ and $\mathbf{r}^{\prime}$, is given by $V\left(\mathbf{r}, \mathbf{r}^{\prime}\right)=e^{2} G\left(\mathbf{r}, \mathbf{r}^{\prime}\right)$, where $G\left(\mathbf{r}, \mathbf{r}^{\prime}\right)$ is the Green's function of the Poisson operator, i.e.,

$$
\boldsymbol{\nabla}_{\mathbf{r}} \cdot \epsilon(\mathbf{r}) \nabla_{\mathbf{r}} G\left(\mathbf{r}, \mathbf{r}^{\prime}\right)=-\delta\left(\mathbf{r}-\mathbf{r}^{\prime}\right)
$$

Therefore, the space dependence of $\epsilon(\mathbf{r})$ modifies $G\left(\mathbf{r}, \mathbf{r}^{\prime}\right)$ with respect to the homogeneous case, where $\epsilon(\mathbf{r})=\epsilon_{\circ}$ and $G_{\circ}\left(\mathbf{r}, \mathbf{r}^{\prime}\right)=1 /\left[4 \pi \epsilon_{\circ}\left|\mathbf{r}-\mathbf{r}^{\prime}\right|\right]$. This, in turn, modifies the Coulomb 
matrix elements between the quantum states of the structure 1 月 wich, in the basis ensuing from the single-particle envelope functions $\Phi^{e\left(e^{\prime}\right)}$, can be written as

$$
V_{i j}=e^{2} \int \Phi_{i}^{e *}(\mathbf{r}) \Phi_{j}^{e^{\prime} *}\left(\mathbf{r}^{\prime}\right) G\left(\mathbf{r}, \mathbf{r}^{\prime}\right) \Phi_{i}^{e^{\prime}}\left(\mathbf{r}^{\prime}\right) \Phi_{j}^{e}(\mathbf{r}) d \mathbf{r} d \mathbf{r}^{\prime}
$$

Here $i, j$ stand for an appropriate set of quantum numbers labelling the states. If the symmetry of the structure is low, as in the realistic quantum wire structures considered below, Eq. (1) must be explicitly solved, and the ensuing potential is then used in (2). In this case it is convenient to cast Eqs. (1) and (2) in Fourier space as described in Ref. 2. In contrast, for particularly simple structures the analytic form of the potential can be directly obtained in real space. This is, e.g., the case for the second prototype structure discussed below, the spherical QD: here two electrons can be shown to interact via the potential

$$
V\left(\mathbf{r}_{i}, \mathbf{r}_{j}\right)=\frac{e^{2}}{\epsilon_{1}} \frac{1}{\left|r_{i}-r_{j}\right|}+\frac{e^{2}}{\epsilon_{1} R_{d}} \sum_{k=0}^{\infty} \frac{(k+1)(\epsilon-1)}{(k \epsilon+k+1)}\left(\frac{r_{i} r_{j}}{R_{d}^{2}}\right)^{k} P_{k}\left(\cos \Theta_{i j}\right),
$$

where $R_{d}$ is the QD radius, $\epsilon=\epsilon_{1} / \epsilon_{2}$, and $\epsilon_{1}\left(\epsilon_{2}\right)$ is the dieletric constant of the inner (outer) material. In the following we discuss the basic results and the relevance of these effects for our prototype QWIs and QDs.

(i) QWIs with remote dielectric confinement.

Recently, we have proposed that remote dielectric confinement (RDC) may be used in order to enhance the exciton binding energies $E_{b}$. In convential nanostructures $E_{b}$ is considerably enhanced by quantum confinement; however, for GaAs-based structures, observed values of $E_{b}$ are still well below $k T_{\text {room }}$. On the other hand, owing to the low optical quality of typical semiconductor/oxide interfaces, oxides cannot be used directly as confining barriers. Our novel approach is based on the idea that quantum and dielectric confinement can be spatially separated since they are effective over different length scales. In the proposed structures the enhanced electron-hole overlap induced by quantum confinement in conventional GaAs/AlGaAs structures is combined with the DC provided by polarization charges which form at a remote interface with a low-dielectric constant material, typically an insulator; since electron and hole wavefunctions decay exponentially into the barrier, they will not be affected by the presumably disordered remote interface.

As an example of our approach, we discuss quantitative predictions for the case of a conventional V-shaped GaAs/AlAs QWI with two oxide layers added above and below the QWI at a distance $L$. The cross-section is shown in Fig. 1(a). The additional layers are characterized by a small dielectric constant that we take equal to 2 (see, e.g., Ref. 6). For this structure, we find $E_{b}=29.3 \mathrm{meV}$, to be compared with $E_{b}=13 \mathrm{meV}$ of the conventional (i.e., with no oxide layers) structure. Fig. 1(a) shows that the origin of this dramatic enhancement is the large polarization of the AlAs/oxide interfaces induced by the excited electron and hole charge densities. A small polarization charge is also induced at the GaAs/AlAs interface, due to the small dielectric mismatch. In Fig. 1(b) we show the calculated $E_{b}$ for selected values of $L$. Obviously, $E_{b}$ is maximum when the oxide layer is at minimum distance $2, L=0$, where it is enhanced by more than a factor 3 with respect to $E_{0}$, and it is well above $k T_{\text {room }}$. The important point here is that $E_{b}$ decreases slowly, indeed as $L^{-1}$, with the distance $L$, and crosses $k T_{\text {room }}$ at $L$ as large as $\sim 9 \mathrm{~nm}$, where the effects of the disorder at the Oxide/AlAs interface are very small.

(ii) Quantum dots in dielectric environments. 

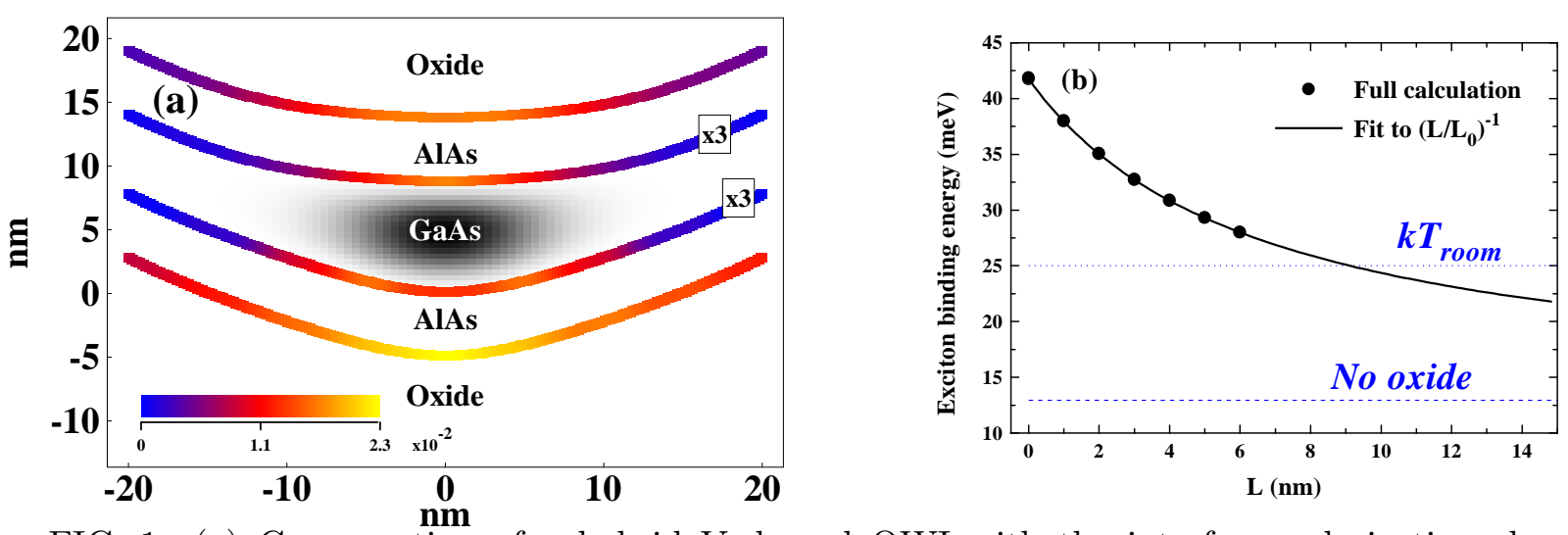

FIG. 1. (a) Cross section of a hybrid V-shaped QWI with the interface polarization charge (units of $\mathrm{nm}^{-1}$ ) and the lowest-subband hole charge density (arbitrary units). The oxide layers are at $L=5 \mathrm{~nm}$ from the inner GaAs/AlAs interfaces. The polarization charge at the GaAs/AlAs interfaces is multiplied by 3 for clarity. (b) $E_{b}$ versus distance of the oxide layers from the inner interfaces. Dots: full calculation. The solid line is a fit to $L^{-1}$. Dashed line: binding energy $E_{0}$ of the corresponding conventional structure (no oxide layers). Dotted line: thermal energy at $T_{\text {room }}=300 \mathrm{~K}$.

These structures have become accessible by transport studies only very recently $\mathrm{O}$ They are III-V or II-VI nanoparticles embedded in materials with different dielectric properties, such as organic matrices in a colloid. QDs in biological environments are also assuming increasing importance. The addition energies $E_{a d d}(N)$ (the energy required to add an electron to a QD containing $\mathrm{N}$ electrons) have been used used to characterize these systems experimentally, but a theoretical description is still lacking. To obtain it, we must compute the ground state energy, $E_{0}(N)$, of the QD with $N$ interacting electrons (assumed to be confined in a spherical parabolic potential). The chemical potential of the QD with $N$ electrons is then $\mu(N)=E_{0}(N)-E_{0}(N-1)$, from which we obtain

$$
E_{\text {add }}(N)=\mu(N+1)-\mu(N)
$$

The ground state energies $E(N)$, obtained from an Hubbard-like approximation to the manybody hamiltoniang, give rise to the addition spectra of Fig. 2. For $N \leq 5$, we have also performed the exact diagonalizations of the many-electron hamiltonian, with results that are almost identical.

The solid line in Fig. 2 is the calculated addition spectrum for a dielectrically homogeneuos QD, i.e., $\epsilon=1$ in Eq. (3). The peaks at $N=2$ and $N=8$ correspond to the addition of one electron to a QD with a closed s- and p-shell, respectively; the weaker peaks at $N=5$ and $N=13$ correspond to the addition of one electron to a QD with a half-filled outer shell where all spins are parallel, as expected by a filling of the shells according to Hund's rule. When $\epsilon>1$ the addition spectra of Fig. 2 are affected in several ways. Let us first consider the behaviour for $N \leq 8$. As $\epsilon$ is increased, the spectra are shifted to higher energies, since a larger energy is needed to add new electrons to the QD due to the enhanced Coulomb repulsion. Note, however, that this shift is not rigid, and the half-shell peak at $N=5$ is enhanced with respect to full-shell peaks at $N=2$ and $N=8$. This result is quite general and derives from the different combinations of direct and exchange Coulomb terms that enter the ground state energies determining the full and half-shell peaks.

The changes taking place at larger $N$ are more dramatic. As $\epsilon$ is increased, the ordering and amplitude of the peaks deviates from the behaviour at $\epsilon=1$. As can be seen Fig. 2, 


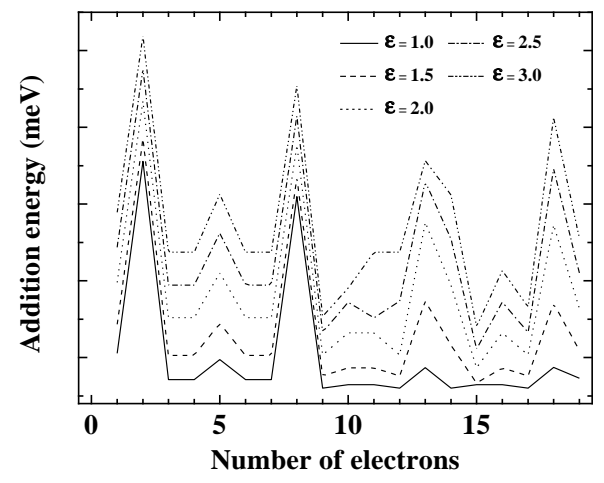

FIG. 2. Addition

spectra obtained with the Hubbard approximation for several values of the dielectric mismatch $\epsilon$. The parameters used in the computations are for a $\mathrm{CdSe} 10$ spherical QD of radius of $3 \mathrm{~nm}$, that corresponds to a confinament energy of about 150 $\mathrm{meV}$.

half-shell peaks become comparable with full-shell ones, and additional features appear for the larger values of $N$. Inspection of the ground state configurations shows that this is due to a shell filling in violation of Hund's rule. Above a critical value $N_{c}$, a reconstruction of the electronic configuration takes place, i.e., the added electron will not be arranged in the most external shell, leaving the remaining electrons in the previous configuration. Instead, it will cause other electrons in the inner shells to be promoted to shells of higher angular momentum. This reconstruction occuring at large values of the dielectric mismatch is similar to the one predicted in QDs in a strong magnetic field (where a similar enhancement of the Coulomb interaction takes place).

In summary, we have shown that dielectric confinement effects may strongly affect quantum states in dielectrically modulated nanostructures. By modulating the dielectric mismatch between different layers it is possible to tune the Coulomb interaction between the quantum confined states, in analogy to what is done by external magnetic fields and/or doping, thereby modifying substantially the optical and addition spectra of nanostructures.

This paper was supported in part by MURST-40\% through grant "Physics of nanostructures" and by INFM through grant PRA-SSQI.

${ }^{1}$ L. V. Keldysh, Pis'ma Zh. Eksp. Teor. Fiz. 29, 716 (1979) [JETP Lett. 29, 658 (1979)].

${ }^{2}$ G. Goldoni, F. Rossi, and E. Molinari, Phys. Rev. Lett. 80, 4995 (1998).

3 The renormalization of the interaction gives also rise to a self-energy term; in most cases this term is small and will not be discussed in this paper. See also Ref.月.

${ }^{4}$ L. E. Brus, J. Chem. Phis., 80, 4403, (1984).

${ }^{5}$ F. Rossi, G. Goldoni, E. Molinari, Phys. Rev. Lett. 78, 3527 (1997) and Refs. therein.

${ }^{6}$ A. Fiore et al., Nature 391, 463 (1998).

${ }^{7}$ Our calculations have been extended down to $L=0$ for completeness, although in this limit they should be taken with caution, due to the neglect of the AlAs/oxide interface effects.

${ }^{8}$ D. L. Klein et al., Nature 389, 6991 (1997).

${ }^{9}$ M. Rontani, F. Rossi, F. Manghi, and E. Molinari, Appl. Phys. Lett. 72, 957 (1998); Phys. Rev. B 59, 10165 (1999).

${ }^{10}$ Landolt-Bornstein, Numerical Data and Functional Relationships in Science and Technology, vol 17, Springer-Verlag, (1982). 